\title{
Design of Realistic Phased Array Patch Elements Using a Genetic Algorithm
}

\author{
Hans Steyskal, Consultant, c/o Sensors Directorate, Air Force Research Laboratory, Hanscom AFB, MA 01731, \\ Tel: (781) 377-2052, Hans.Steyskal.ctr@hanscom.af.mil
}

\begin{abstract}
Scott Santarelli, Sensors Directorate, Air Force Research Laboratory, Hanscom AFB, MA 01731, Tel: (781) 377-6854,
Scott.Santarelli@hanscom.af.mil
\end{abstract}

\begin{abstract}
Presently most wide band array elements are complicated 3-dimensional structures which are difficult and costly to manufacture. Therefore we explore planar patch elements which offer a simplified geometry with potentially reduced weight and cost and are suitable for conformal applications. We use a genetic algorithm to design a patch element in a unit cell of an infinite periodic array and model the feed with the usual $\delta$-gap voltage source. However, since this feed is a non-realizable mathematical concept, we also explore the antenna performance achieved with several realistic feeds. For broadside arrays we obtain a bandwidth $B \approx 4: 1$ both with the $\delta$-gap and with the realistic feeds. For arrays with scan angles out to $45^{\circ}$, the initial bandwidth $B \approx 2: 1$ with the $\delta$ gap is reduced to $B \approx 1.7: 1$ with a differential coaxial feed, whereas the initial bandwidth is maintained with a GAoptimized custom feed. The element profiles range from 0.13 $\lambda_{L}$ to $0.17 \lambda_{L}$, where $\lambda_{L}$ denotes the wavelength at the low end of the operational band.
\end{abstract}

\section{INTRODUCTION}

This research concerns the continued development of wide band, wide scan, low profile phased arrays for aircrafts and UAVs. Presently most wide band array elements are complicated 3-dimensional structures which are difficult and costly to manufacture [1-4]. As an alternative, we explore planar patch elements which offer a simplified geometry with potentially reduced weight and cost and are suitable for conformal applications.
Our earlier research has resulted in planar elements with more than octave bandwidth and scan range up to $45^{\circ}$ off broadside [5-6]. The approach was inspired by [7] and is based on a genetic algorithm (GA) that optimizes the patch design. The designs assumed an idealized mathematical feed, a $\delta$-gap voltage source, as is common in theoretical antenna studies. Unfortunately, however, such sources do not exist and their replacement by a realistic feed may seriously degrade the array performance. Therefore, in this paper we explore the effects of realistic feeds using two different methods. For a given GA design of a fragmented patch with a $\delta$-gap feed we 1) replace the $\delta$-gap feed with one of several known real feeds, or 2) design a custom feed using the GA.

\section{The ApProACH}

Our approach uses a genetic algorithm to design a patch element in a unit cell of an infinite periodic array. Fig. 1 shows top and side views of the unit cell, which includes sub- and superstrates of variable thickness $d$ and relative permittivity $\varepsilon$. The patch, which is fed at its center by a $\delta$ gap voltage source, is 'fragmented' into a set of pixels that are either conducting or non-conducting. A GA determines the pixel distribution and the sub/superstrates for optimal wide band and wide scan performance by minimizing a cost function $F$ based on reflected power:

$$
F=\sum_{i} \sum_{j} w_{i j}\left|R\left(\Omega_{i}, f_{j}\right)\right|^{4},
$$

where $R$ denotes the active reflection coefficient, $\Omega_{i}$ the sample scan angles, $f_{j}$ the sample frequencies over the design band, and $w_{i j}$ are heuristically chosen weights.

We use the exponent 4 rather than the standard 2 exponent in order to achieve a more uniform, low-level reflection coefficient over the frequency band. For the evaluation of $\Gamma\left(\Omega_{i}, f_{j}\right)$ we use a highly efficient FDTD code [8], which in one run produces the wide band response of the 
Top View

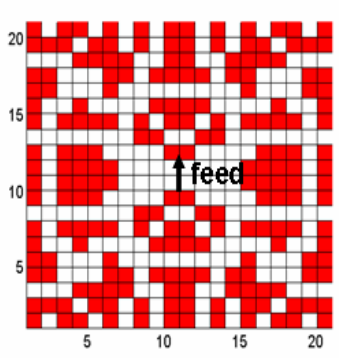

Side-View

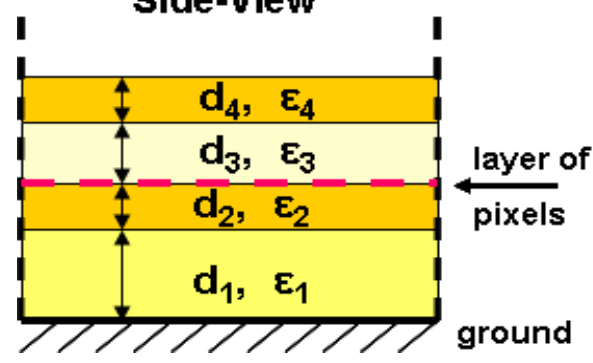

Figure 1--Horizontal and vertical cross sections of array unit cell with two sub- and two superstrates of thickness $d_{i}$ and rel. permittivity $\varepsilon_{\mathrm{i}}$. Conducting pixels are shown in red.

array element for any particular scan angle.

It is desirable to work with reasonably high resolution, which, however, must be traded off against computation time. For example, fragmenting the aperture into $20 \times 20$ pixels corresponds in principle to a set of $2^{400}$ different possible geometries. Imposing symmetry among the four quadrants reduces this number to $2^{100}$, which still represents an extremely large number of alternatives for the GA to search through. Moreover, we have found that we need to model each pixel by $4 \times 4$ fdtd-cells to maintain numerical accuracy. Both the large number of possible geometries and the large number of fdtd-cells contribute to increases in the computation time. Thus, to keep the computation time practical, i.e. less than a week, we normally use only $10 \times 10$ pixels.

\section{FEED TYPES}

The various feed types which we considered are shown in Fig. 2. The $\delta$-gap voltage source with an internal resistance $\mathrm{R}_{\mathrm{i}}$ that matches the antenna radiation resistance,(see Fig. $2 \mathrm{a}$ ), is the most convenient feed for theoretical antenna analysis (and for publishing papers). Unfortunately, it is only an idealized concept and cannot be realized in practice.

The simplest realistic feed is the single coaxial line shown in Fig. 2 b, where the inner and outer conductors connect to the terminals on the patch. The characteristic impedance of the coaxial line again has to match the antenna impedance. However, it represents an 'unbalanced' feed and thus there will be currents flowing on the vertical outer conductor, which will affect the performance.
A better alternative is the differential coaxial feed, (seeFig. $2 \mathrm{c}$ ), where two coaxial lines excite the patch with signals of equal amplitude and $180^{\circ}$ phase difference. This represents a balanced feed and for broadside scan there will be no current (no 'common' mode) induced on the vertical outer conductors. For off-broadside scan, i.e. for asymmetric excitation, both the 'differential' mode and the 'common' mode will be excited. A $180^{\circ}$ four-port hybrid as shown in Fig. 2c provides a practical way to generate the differential feed excitation. We chose to leave the $\Sigma$-port open-circuited (reflection coeff. $=1$ ) which causes the terminal currents on the opposing side to be equal in magnitude and opposite in phase, regardless of the antenna impedance [9]. In this case, the currents will be balanced, and no common mode current will flow.

Another alternative is the twin-line feed, shown in Fig. 2d. The dimensions of the line have to be chosen such that its characteristic impedance matches that of the array element. The hybrid is again a convenient way to excite the twin-line from an unbalanced, single input.

Finally, in Fig. 2e we show a 'custom' feed, where we let the GA design the feed structure that provides a transition to the patch from a single coaxial input at the ground plane. The basic structure contains two 'shorts' from the patch terminals down to the ground plane, and a center conductor which connects to one of the patch terminals. The widths of these conductors and their spacings are optimized by the GA. 


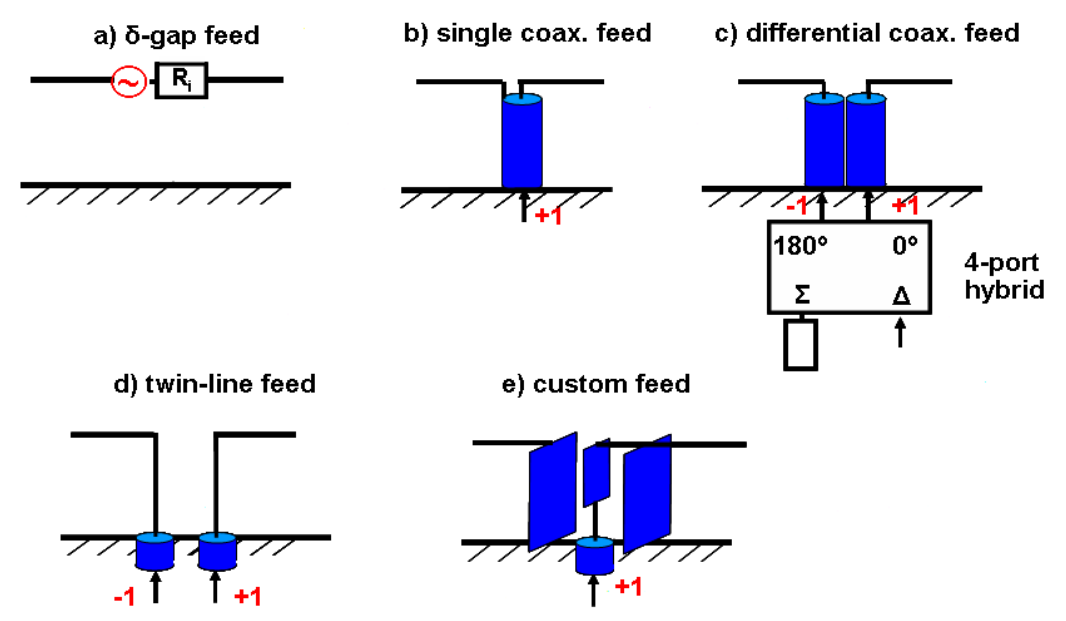

Figure 2 -- Different feed types

\section{RESUlts}

The unit cell size in all the following examples is $30 \times 30$ $\mathrm{mm}$, corresponding to $\lambda / 2$ at $5 \mathrm{GHz}$. The bandwidth is conservatively defined as the frequency band over which the active reflection coefficient $\mathrm{R}<-10 \mathrm{~dB}$.

\section{Patches with $\delta$-Gap Feeds}

We first tested the approach on a 'broadside patch', i.e. the design of a wideband patch with a $\delta$-gap feed at broadside scan. This resulted in the patch design shown in Fig. 3 and sub- and superstrate dimensions $d_{1}=14.25 \mathrm{~mm}, \varepsilon_{1}=1, d_{2}=1.5$ $\mathrm{mm}, \varepsilon_{2}=3.4, d_{3}=7.5 \mathrm{~mm}, \varepsilon_{3}=2.6, d_{4}=0$. The desired bandwidth is $2.0-8.0 \mathrm{GHz}$ in this case and the realized bandwidth is $1.8-8.1 \mathrm{GHz}$. The total profile is $\approx 0.15 \lambda_{\mathrm{L}}$ at the low end of the operational band. Repeated runs with increased desired bandwidth did not result in a correspondingly increased realized bandwidth, which presumably was due to the limited resolution of only 10 pixels.

A second example was a 'wide scan patch' with a desired

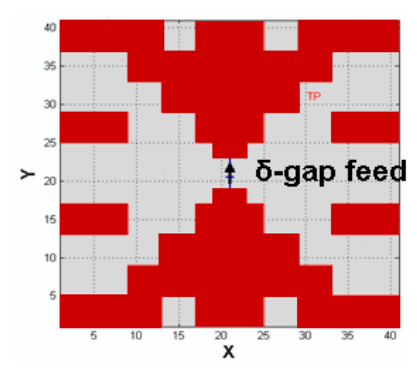

bandwidth $2.0-4.5 \mathrm{GHz}$ and scan out to $45^{\circ}$ in both the Eand H-plane. The resultant design is shown Fig. 4, and the sub- and superstrate dimensions are $d_{1}=17.5 \mathrm{~mm}, \varepsilon_{1}=1.1$, $d_{2}=1.5 \mathrm{~mm}, \varepsilon_{2}=3.4, d_{3}=\mathrm{d}_{4}=0$. The patch has a realized bandwidth of $2.0-4.3 \mathrm{GHz}$. and a profile $\approx 0.13 \lambda_{\mathrm{L}}$ at the low end of the operational band.

\section{Patches with Real Feeds}

We excited the 'broadside patch' with a single coaxial line feed, a differential coaxial feed and a twin-line feed. In all cases the feed was matched to the $200 \mathrm{ohm}$ impedance of the patch. The active reflection coefficient corresponding to these three cases is shown in Fig. 5. Not too surprisingly the unbalanced single coaxial feed does not perform well and in this case reduces the initial bandwidth from $1.8-8.1 \mathrm{GHz}$ to $3.0-5.5 \mathrm{GHz}$. The other two feeds incur practically no bandwidth reduction.

Similarly we excited the 'wide scan patch' with the same set

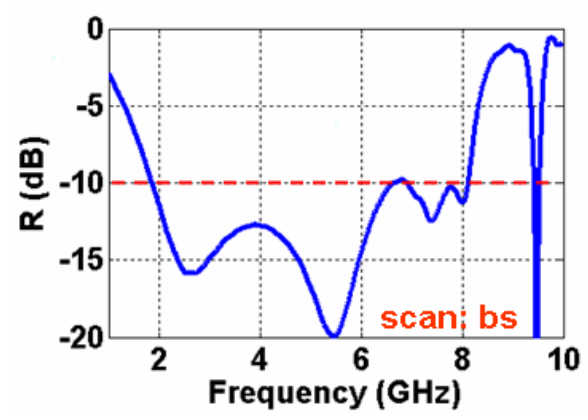

Figure 3 -- The broadside patch design with $\delta$-gap feed and the active reflection coefficient vs. frequency. 
of feeds, the single coaxial line feed, the differential coaxial feed and the twin-line feed, which in this case were matched to the $120 \mathrm{ohm}$ impedance of the patch.

The resultant active reflection coefficient vs. frequency and scan angle are shown in Fig. 6 . We note that the bandwidth reduction can be quite dramatic in this case. The single coaxial feed even at broadside scan reduces the bandwidth by about $35 \%$, and considering all three scan angles, the bandwidth is actually reduced to zero.

The two other feeds lead to roughly equal performance with a realized bandwidth of $2.0-3.4 \mathrm{GHz}$, the bandwidth reduction occurring for the E-plane scan. For scan in the $\mathrm{H}$ plane, where the coupling is relatively low, the common mode is not strongly excited and the balanced feeds work well.

\section{Patch with Custom Feed}

Finally we show one example of a custom feed which resulted from a GA optimization of the shapes and spacings of the conductors comprising the feed. This patch was somewhat different than the previous wide scan patch. The patch geometry is shown in Fig. 7a, the sub-and superstrate dimensions and permittivities are $d_{1}=14.25 \mathrm{~mm}, \varepsilon_{1}=1.2$, $d_{2}=1.5 \mathrm{~mm}, \varepsilon_{2}=3.4, d_{3}=7.5 \mathrm{~mm}, \varepsilon_{3}=1.0, d_{4}=2.25 \mathrm{~mm}, \varepsilon_{4}=6.4$, and the impedance is $200 \mathrm{ohm}$. With the $\delta$-gap feed the realized bandwidth is $2.1-4.4 \mathrm{GHz}$, see Fig. 7b. With the custom feed as shown in Fig. 8a, the realized bandwidth is $2.0-4.0 \mathrm{GHz}$, see Fig. 8b. This is only a very small bandwidth reduction which, however, is achieved with a rather complicated feed geometry. - The computation time for this case was about 30 days.
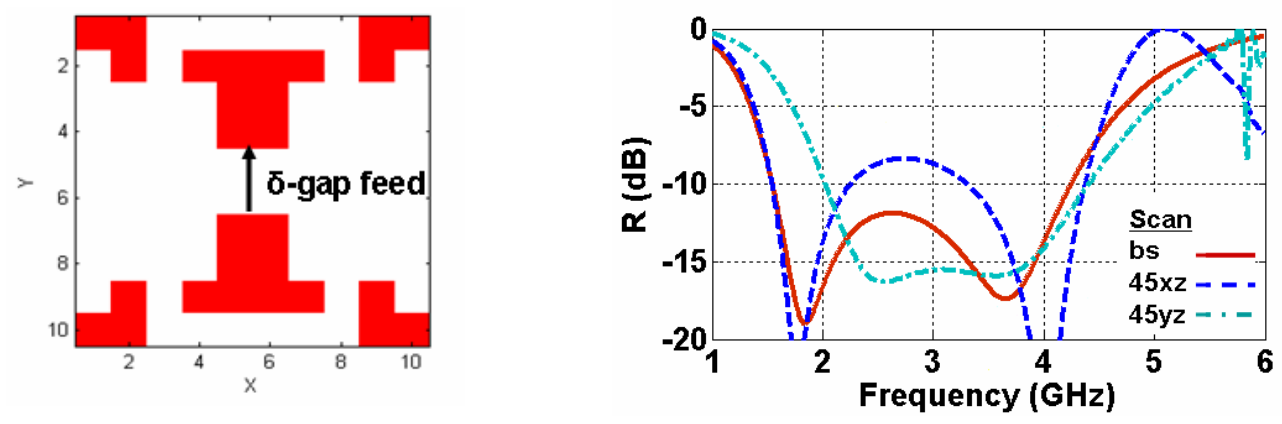

Figure 4-- The wide scan patch design with $\delta$-gap feed and the active reflection coefficient vs. frequency ("bs", “45xz", and "45yz" denote scan angles of broadside, 45 deg. xz- and 45 deg. yz-plane, resp.).
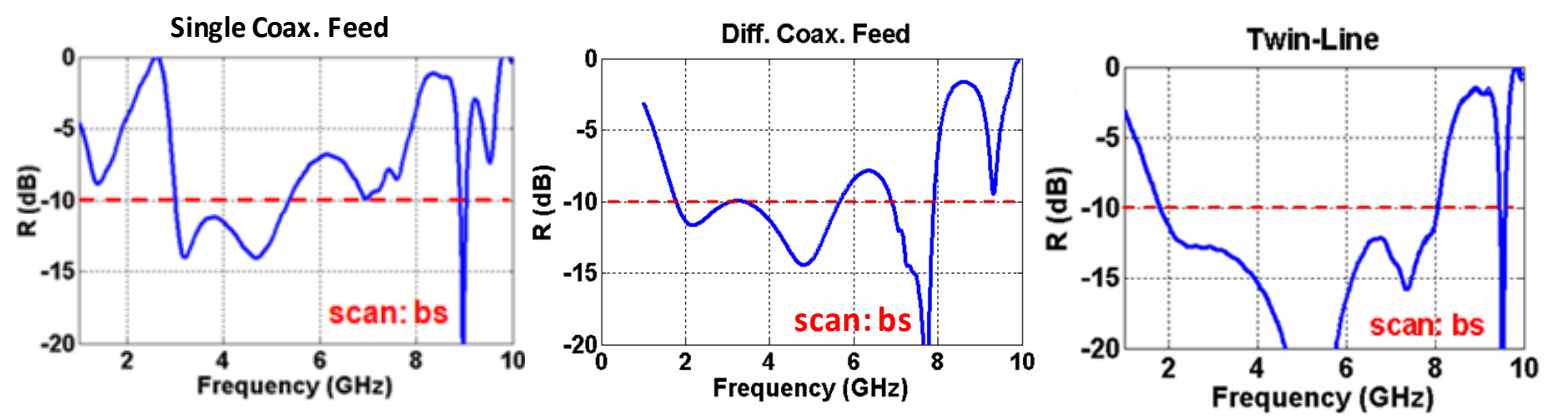

Figure 5--The broadside patch with different feeds - active reflection coefficient vs. frequency. 

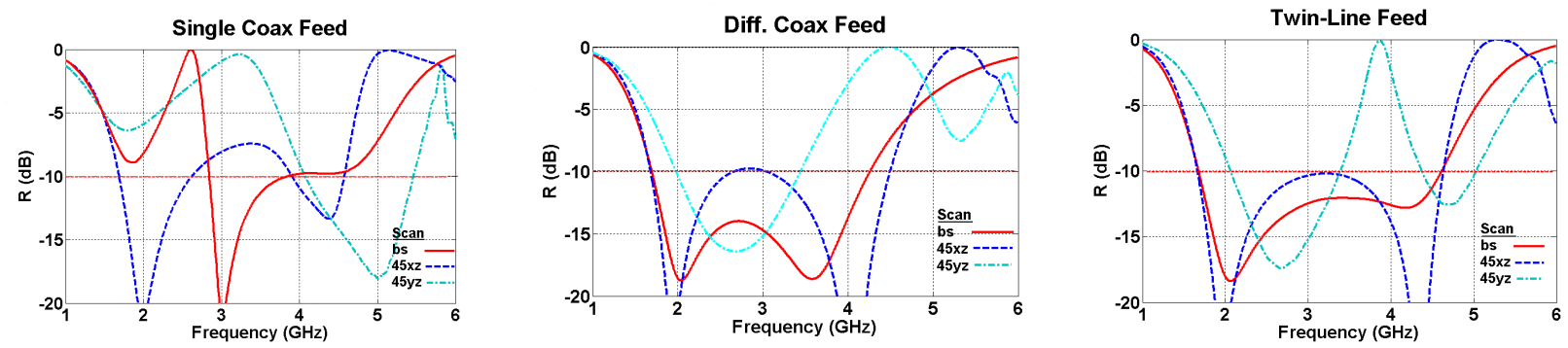

Figure 6--The wide scan patch with different feeds - active reflection coefficient vs. frequency ("bs", "45xz", and “45yz" denote scan angles of broadside, 45 deg. xz- and 45 deg. yz-plane, resp.).

(a)

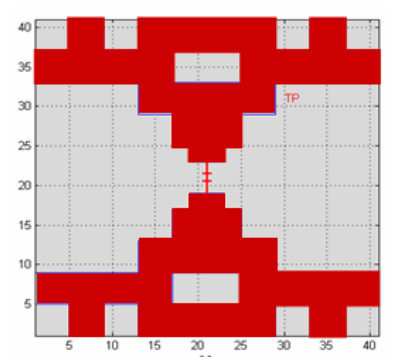

(b)

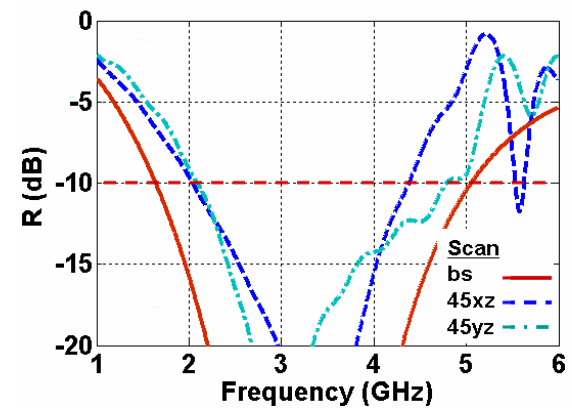

Figure 7-- Wide scan patch with $\delta$-gap feed and active reflection coefficient vs. frequency.
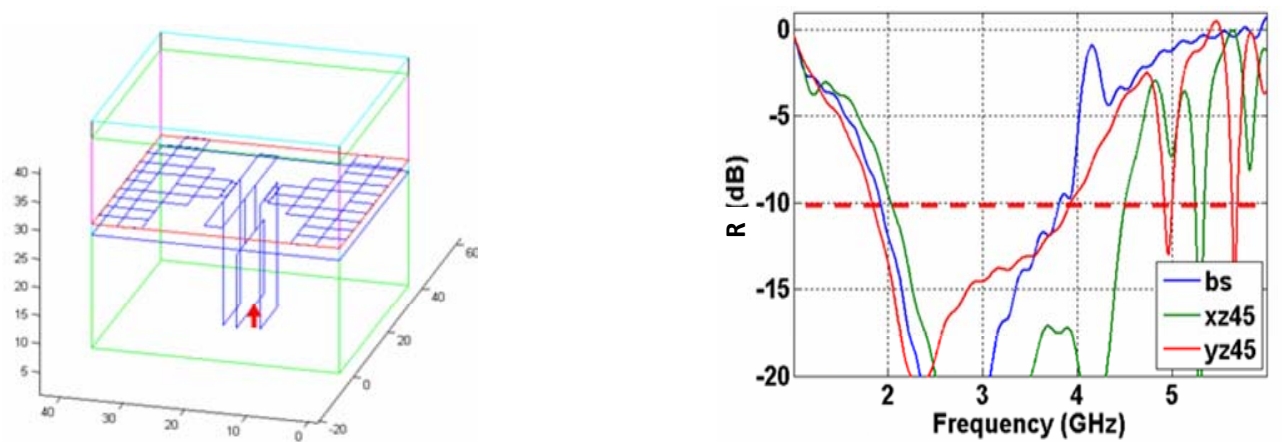

Figure 8-- Wide scan patch from Fig. 7 with GA designed custom feed and corresponding active reflection coefficient vs. frequency.

\section{SUMMARY AND CONCLUSION}

For the different patch elements the above results of bandwidth vs. feed type are summarized in Table 1.

For the broadside patch, with a design frequency band 2.0$8.0 \mathrm{GHz}$, the desired $\mathrm{B}=4: 1$ bandwidth is achieved both with the $\delta$-gap feed and with realistic feeds. The profile of this element is $\approx 0.15 \lambda_{\mathrm{L}}$ at the low end of the operational band.
For wide angle scanning arrays, the design of a realistic feed, which avoids a reduction of the desired 2.0-4.5 GHz band, clearly poses a difficult problem. Patch 1 achieves an initial bandwidth $\mathrm{B}=2: 1$ with a $\delta$-gap feed which is reduced to $\mathrm{B}=1.7: 1$ with a twin-line feed. This element has a profile $\approx 0.13 \lambda_{\mathrm{L}}$ at the low end of the operational band. Patch 2 also has an initial bandwidth $B \approx 2: 1$, which is practically maintained with the GA designed custom feed. The profile of this latter element is $\approx 0.17 \lambda_{\mathrm{L}}$ at the low 
Table 1-- Bandwidth vs. Feed Type for Different Patch Elements

\begin{tabular}{|l|c|c|c|}
\hline Feed & Broadside Patch & Wide Scan Patch 1 & Wide Scan Patch 2 \\
\hline$\delta$-gap & $1.8-8.1 \mathrm{GHz}$ & $2.2-4.3 \mathrm{GHz}$ & $2.1-4.4 \mathrm{GHz}$ \\
\hline single coaxial & $3.0-5.5 \mathrm{GHz}$ & 0 & --- \\
\hline differential coaxial & $1.8-5.7 \mathrm{GHz}$ & $2.0-3.4 \mathrm{GHz}$ & --- \\
\hline twin-line & $1.8-8.0 \mathrm{GHz}$ & $2.1-3.4 \mathrm{GHz}$ & --- \\
\hline custom & --- & -- & $2.0-4.0 \mathrm{GHz}$ \\
\hline
\end{tabular}

end of the operational band. Thus it has a somewhat higher profile than the first element; however, it does not require the 4-port hybrid.

The antenna performance of our fragmented patch elements is presently probably limited by our simple geometry and improvements might be obtained with higher resolution, more inter-element coupling control, and additional frequency selective surfaces. An obvious alternative also is to include both the patch and the feed structure in the GA optimization, and such attempts are under way. However, the increased number of free parameters leads to rather long computation times.

\section{ACKNOWLEDGEMENT}

This study was supported by the US Air Force Office of Scientific Research under Dr. Arje Nachman.

\section{REFERENCES}

[1] H. Holter, T-H Chio, D. Schaubert, "Elimination of Impedance Anomalies in Single- and Dual-Polarized EndFire Tapered Slot Phased Arrays," IEEE Trans AP, Jan. 2000

[2] D. McGrath, "Numerical Analysis of TEM Horn Arrays," Sensor and Simulation Notes, No. 420, AFRL, Albuquerque, May, 1998.

[3] J.J. Lee, S. Livingstone, R. Koenig, "A Low-Profile Wide-Band (5:1) Dual-Pol Array," IEEE Antennas and Wireless Propagation Letter, Vol. 2, p. 46, 2003

[4] H. Holter, "A New Type of Antenna Element for WideBand Wide-Angle Dual Polarized Phased Array Antennas," IEEE Int'l Symp. on Phased Array Systems and Technology, Boston, MA, Oct. 2003

[5] B. Thors, H. Steyskal, H. Holter, "Broadband Fragmented Aperture Phased Array Element Design Using Genetic Algorithms," IEEE Trans-AP, Oct. 2005

[6] H. Steyskal, D. Hanna, "Design Aspects of Fragmented Patch Elements for Phased Arrays," IEEE AP-S Int'1 Symp.,
Honolulu, Hawaii, June 2007

[7] P. Friedrich et al, "A New Class of Broadband Planar Apertures," Antenna Application Symp., Allerton Park, IL, Nov. 2001

[8] H. Holter, H. Steyskal, “ Infinite Phased Array Analysis Using FDTD Periodic Boundary Conditions - Pulse Scanning in Oblique Directions," IEEE Trans. AP, Oct. 1999

[9] J. S. McLean, "Balancing Networks for Symmetric Antennas - 1: Classification and Fundamental Operation," IEEE Trans. Electromagnetic Compatibility, No. 4, Nov. 2002

\section{BIOGRAPHY}

Hans Steyskal received the degrees Civ. Ing., Tekn. Lic., and Tekn. Dr. in electrical engineering from the Royal Institute of Technology (KTH), Stockholm, Sweden in 1963, 1970 and 1973, respectively. In 1962, he joined the National Defence Research Establishment (FOA), where he investigated microwave radiation and scattering problems. In 1980, he left his position as Chief, Section for Field and

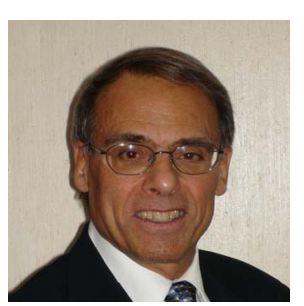

Circuit Theory, and moved permanently to the United States. There he has pursued his research interests in electromagnetics, phased array antennas, digital beamforming and array signal processing at the $A F$ Research Laboratory, Antenna Technology Branch, Hanscom AFB, MA, since 2004 as AFRL Senior Scientist for Antennas. In 2007 he retired and works now as an AFRL on-site contractor.

Dr. Steyskal has been a part-time Adjunct Professor in Antenna Technology at KTH during 1996-2004, and a Visiting Scientist at the Polytechnic University of New York and at The Federal Institute of Technology, Lausanne, Switzerland, and has served two terms as Associate Editor for the IEEE Transaction on Antennas and Propagation. He is an AFRL Fellow and a Fellow of the IEEE. 
Scott Santarelli was born on March 22, 1970 in Haverhill, MA, USA. He obtained a B.S. in Electrical Engineering from the University of Lowell in 1992, and a M.S. in Electrical Engineering with a concentration in Fields and Waves from Northeastern University in 1995. He received his Ph.D. in Cognitive and Neural Systems from Boston University in 2001. His research interests include the study of irregularly-shaped subarray architectures for planar arrays, the study of frequency-selective surfaces for infinite, periodic arrays, and the application of genetic algorithms and neural network concepts to antenna design and optimization. 